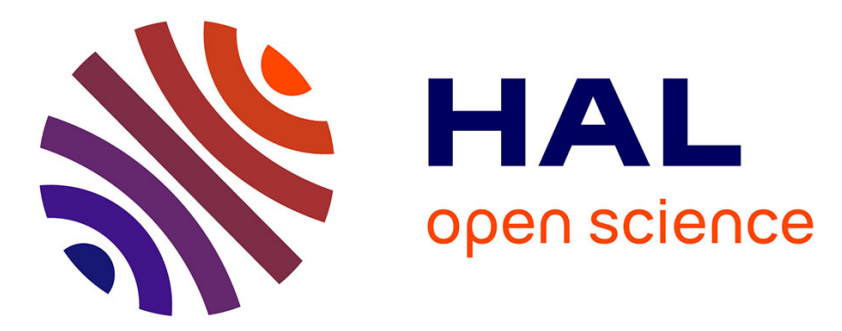

\title{
Transport barriers associated to the resonant interaction between trapped particle modes triggered by plasma polarization injection
}

\author{
A. Ghizzo, D. del Sarto, F. Palermo, A. Biancalani
}

\section{- To cite this version:}

A. Ghizzo, D. del Sarto, F. Palermo, A. Biancalani. Transport barriers associated to the resonant interaction between trapped particle modes triggered by plasma polarization injection. EPL - Europhysics Letters, 2017, 119 (1), 10.1209/0295-5075/119/15003 . hal-01791762

\section{HAL Id: hal-01791762 \\ https://hal.univ-lorraine.fr/hal-01791762}

Submitted on 14 May 2018

HAL is a multi-disciplinary open access archive for the deposit and dissemination of scientific research documents, whether they are published or not. The documents may come from teaching and research institutions in France or abroad, or from public or private research centers.
L'archive ouverte pluridisciplinaire HAL, est destinée au dépôt et à la diffusion de documents scientifiques de niveau recherche, publiés ou non, émanant des établissements d'enseignement et de recherche français ou étrangers, des laboratoires publics ou privés. 


\title{
Transport barriers associated to the resonant interaction between trapped particle modes triggered by plasma polarization injection
}

\author{
A. Ghizzo ${ }^{1}$, D. Del Sarto ${ }^{1}$, F. Palermo ${ }^{2}$, A. Biancalani ${ }^{2}$ \\ 1 Institut Jean Lamour- UMR 7168, University of Lorraine, BP 239 F-54506 Vandoeuvre les Nancy, France. ${ }^{2}$ Max \\ Planck Institute for Plasma Physics, Germany
}

\author{
PACS 52.65.Tt - Gyrofluid and gyrikinetic simulations \\ PACS 52.25.Dg - Plasma kinetic equations \\ PACS 52.35.Ra - Plasma turbulence
}

\begin{abstract}
The Letter explores the parametric excitation of low-frequency zonal flow induced by the beating of collisionless trapped ion and trapped electrons modes (CTIMs and CTEMs) and its feedback on the formation of transport barrier (TB). The zonal flow generation and the associated intermittent transport are investigated using a semi-Lagrangian gyrokinetic Vlasov simulations based on a Hamiltonian reduction technique, where both the fastest scales (cyclotron and bounce motions) are gyro-averaged. The model confirms the possibility to trigger TB by means of polarization effects, recently observed in global gyrokinetic simulations by A. Strugarek et al, Phys. Rev. Lett. 111 (2013) 145001.
\end{abstract}

Introduction. - Motivated by the experimental discovery of the low-to-high (LH) transition 1 in the plasma confinement regimes, experiments and theory in the last decade have focused on whether the turbulence associated with the H- states 2] may be regulated by interactions with zonal flows (ZFs). Central to all the enhanced confinement regimes, be they in the edge region or in the core region of the tokamak plasma, is the generation of ZFs and sheared flows, which are believed to be responsible for suppressing fluctuations and stabilising the turbulence. They are also key ingredients to trigger bifurcations towards the generation of transport barriers (TBs) [3] which are essential for elements in reference scenarii for thermonuclear plasma confined in tokamak devices [4].

A clear indication of the key role played by ZFs was the recent observation at the Experimental Advanced Superconducting Tokamak (EAST) of a low-frequency signal at a few kilohertz attributed to oscillating ZFs [5]. The signal was observed at a much lower frequency than the geodesic acoustic modes (GAMs). No coherent oscillations in the GAM frequency range was detected by the probes in these experiments in the $\mathrm{H}$-mode regime. These experimental measurements show strong correlations with trapped ion population, while standard GAMs are not usually observed in these regimes (we can then assume that circulating particles do not play here a significant role). Recently the feedback of turbulence- driven stream- ers on ZF stability has been investigated in Ref. [7] where the occurrence of such low frequency regime of oscillations for ZFs has been shown. In principle low-frequency ZFs may occur in the core of the plasma. Indeed both (low -frequency and zero -frequency) components of ZFs have been observed in the DIII-D tokamak in a frequency broadband $(0-10 \mathrm{kHz})$ in 6], together with the high- frequency GAM component.

In contrast, streamers contribute to enhance the transport owing to their radial elongated structures. Streamers, observed in numerical simulation studies, appear to be closely associated with avalanche type transport events. They can be produced in the saturation regime of trapped ion modes [7. Collisionless trapped ion modes (CTIMs) are known to be a prototype of kinetic modes since they are driven through the resonant interaction between a wave and trapped ions through their precession motion. CTIMs have been studied by Kadomtsev and Pogutse 40 year ago (see Ref. [8]), and are known to be subject to interchange- type turbulence. Recently [9], it was shown that trapped ions can also resonate with the low drift precession and thus enhance the neo-classical toroidal viscosity, which expresses a modification of the radial transport caused by non axisymmetric magnetic perturbations.

While substantial effort has been devoted to the study of ZF generation by drift-wave or Ion Temperature Gradi- 
ent (ITG) modes, little work has been published on zonal flows driven by pure collisionless trapped ion modes or by their electron counterpart, the collisionless trapped electron modes (CTEMs) when electron dynamics is taken into account. Recently in Refs 11, 12, we have identified a nonlinear coupling mechanism of $\mathrm{ZF}$ mediated by CTIMs in the collisionless regime, leading to a non-zero low-frequency ZF mode of resonant nature (with the possibility to an amplification by wave- "banana" resonance). While zero- frequency ZFs are quite non resonant, being relativily easy to drive up by the Reynolds tensor, this new low-frequency oscillating component of $\mathrm{ZF}$ becomes sensible to a strong amplification induced by the resonant interaction between CTIM and CTEM. Thus this low-frequency mode plays a major role in the dynamics interaction among ZFs, streamers, shear flow and interchangetype turbulence, which are usually implicated in the low- high ( $\mathrm{LH})$ transition. In this letter, we study the role of the low-frequency $\mathrm{ZF}$ component, which can trigger such a transition by regulating the turbulence, provided its amplitude is high enough. The different steps are investigated: from a simplified predator- prey picture of the LH transition including this new ZF component, till the parametric amplification described by a trapped particle model where the low- frequency $\mathrm{ZF}$ leads to a bursty turbulent behavior. Finally we report on the observation of TB generation in simulation of ITG driven turbulence (but restricted to kinetic CTIMs), when resonance with CTEMs is triggered by plasma polarization fluctuations. Numerical simlations have been carried out in a regime of improved core confinement where an (internal) barrier transport was observed when $T_{e} \leq T_{i}$ (see for instance Fig. 75 of the plasma review [10]).

The trapped particle mode description. - We choose to start with the trapped particle model of kinetic nature, while the predator- prey model will be recall later. By describing the phase space in action angle $(\psi, \alpha)$ coordinates system $\left(\alpha=\varphi-q_{0} \theta\right.$ being the precession angle and $\psi$ the poloidal flux), the population of trapped particle modes is here described by a distribution function $f_{s}=f_{s ; E, \kappa}(\psi, \alpha, t)$ for each particle species $(s=e, i$ for electrons and ions respectively) and where $E$ and $\kappa(\kappa<1)$ are considered as adiabatic invariants. The low-frequency response for CTIMs is obtained by making a phase-angle average over the cyclotron phase and the bounce motion (the "banana" orbit) leading to invariance of the total energy $E_{s}=\frac{1}{2} m_{s} v_{G \|}^{2}+\mu B\left(\boldsymbol{x}_{G}\right)$ and of the so-called adiabatic invariant $\mu=\frac{m_{s} v_{G}^{2}}{2 B\left(\boldsymbol{x}_{G}\right)}$. Here the label $G$ is a conventional notation which refers to the guiding center and $\boldsymbol{x}_{G}$ refers to $(r, \theta)$ polar coordinates. By introducing the pitch angle parameter $\kappa$ defined by $\kappa^{2}=\sin ^{2}\left(\frac{\theta_{0}}{2}\right)=\frac{1-\lambda}{2 \varepsilon \lambda}$ where $\lambda=\frac{\mu B_{0}}{E}$ and $\varepsilon=\frac{r}{R_{0}}$ is the inverse aspect ra- tio. Following the work of Kadomtsev and Pogutse [8] the bounce frequency is given by $\omega_{b s}=\sqrt{\frac{2 E_{s}}{m_{s}}} \frac{1}{q_{0} R_{0}} \bar{\omega}_{b}(\kappa)$ with $\bar{\omega}_{b}(\kappa) \simeq \frac{\pi \sqrt{\varepsilon}}{2 \sqrt{2} K(\kappa)}$. The corresponding precession frequency is then $\omega_{d s}=\frac{q_{0} E_{s}}{e r_{0} R_{0} B_{0}} \bar{\omega}_{d}(\kappa ; s)$ with $\bar{\omega}_{d}(\kappa ; s)=$ $\frac{2 E(\kappa)}{K(\kappa)}-1+4 s\left(\frac{E(\kappa)}{K(\kappa)}+\kappa^{2}-1\right)$. Here $s=\frac{r_{0}}{q_{0}}\left(\frac{d q}{d r}\right)_{r_{0}}$ is the magnetic shear and $K(\kappa)$ and $E(\kappa)$ are the complete elliptic integrals of the first and second kind respectively. The resulting gyrokinetic Vlasov equations is then given by:

$$
\frac{\partial f_{i}}{\partial t}+\omega_{d}(\kappa) \frac{E_{i}}{T_{0}} \frac{\partial f_{i}}{\partial \alpha}+\left[J_{0 i} \phi, f_{i}\right]=\partial_{\psi}\left(D(\psi) \partial_{\psi} f_{i}\right)
$$

and

$$
\frac{\partial f_{e}}{\partial t}-\omega_{d}(\kappa) \frac{E_{e}}{T_{0}} \frac{\partial f_{e}}{\partial \alpha}+\left[J_{0 e} \phi, f_{e}\right]=\partial_{\psi}\left(D(\psi) \partial_{\psi} f_{e}\right)
$$

where $[.,$.$] is the usual Poisson bracket defined by [g, f]=$ $\partial_{\psi} g \partial_{\alpha} f-\partial_{\alpha} g \partial_{\psi} f$ and $J_{0 s} \phi$ is the bounce average electric potential for the species $s$ and the gyro-average operator $J_{0 s}$ was approximated by the Pade's relation in the following form:

$$
J_{0 s}=\left(1-\frac{E_{s}}{4 T_{s}} \delta_{b s}^{2} \partial_{\psi}^{2}\right)^{-1}\left(1-\frac{E_{s}}{4 T_{s}} \frac{\rho_{c s}^{2}}{a^{2}} \partial_{\alpha}^{2}\right)^{-1}
$$

Here $\delta_{b s}$ is constant (we have neglected the dependence in $\kappa)$ and is found to have a value close to $\frac{\rho_{c s} q_{0}}{\sqrt{\varepsilon}}$ where $\rho_{c s}$ is the Larmor radius of species $s$. Assuming that $\frac{\rho_{c s}}{a}=$ $\rho^{*} \sqrt{\frac{T_{s} m_{s}}{T_{i} m_{i}}}$ with $\rho^{*}=\frac{\rho_{c i}}{a}$, the Vlasov eqs. (11) and (2) are then coupled using the quasi-neutrality condition $\delta n_{e}=$ $\delta n_{i}$ which reads as:

$$
C_{e} \frac{T_{0}}{T_{i}}\left(\phi-\langle\phi\rangle_{\alpha}\right)-C_{i} \frac{T_{0}}{T_{i}}\left(1+\frac{m_{e}}{m_{i}}\right) \bar{\triangle} \phi=n_{i}-n_{e}
$$

where

$$
\bar{\triangle} \equiv q_{0}^{2} \rho^{* 2} \partial_{\alpha}^{2}+\delta_{b s}^{2} \partial_{\psi}^{2}
$$

The particle density is defined by

$$
n_{s}(\psi, \alpha, t)=\int_{0}^{1} d \kappa \kappa K(\kappa) \frac{2}{\sqrt{\pi}} \int_{0}^{+\infty} d E_{s} \sqrt{E_{s}} J_{0 s} f_{\kappa, E}
$$

Here $C_{e}$ and $C_{i}$ are constants accounting for the fraction of trapped particles $f_{p}$ and for the ratio $\tau=\frac{T_{i}}{T_{e}}$ of ion to electron temperatures $C_{e}=(1+\tau) / f_{p}$ and $C_{i}=C_{e} f_{p} / \tau$. Thus trapped ion turbulence develops on length scale of the order of the banana width $\delta_{b s}$ and time scale determined by $\omega_{d 0}^{-1}$ where $\omega_{d 0}=\frac{q_{0} T_{0}}{e r_{0} R_{0} B_{0}} . B_{0}$ is the minimal value of the magnetic field amplitude $B$ at $\theta=0\left(R=R_{0}\right.$ being then the major radius). 
Kinetic aspect of ZFs driven by the interchange turbulence. - Usually ZFs are non resonant with respect to wave-particle interactions since they can be nonlinearly generated by the Reynolds tensor. While ZFs backreact upon turbulence by vortex shearing, weakening the source of the turbulence, shear flows damp and then break up ZFs. This latter mechanism is however weakened by the modification of the nature of ZFs, in presence of interchange-type turbulence, in particular in the resonant regime of CTIMs. It is possible to introduce a "mean" pressure in the form $P_{s}=\left\langle P_{s}\right\rangle_{\alpha}+\delta P_{s}\left(\right.$ with $\left.\left\langle\delta P_{s}\right\rangle_{\alpha}=0\right)$ which plays a role analogous to the zonal flow component $\langle\phi\rangle_{\alpha}$. In the framework of the trapped-ion model, we have obtained the equations of the mean potential fluctuations $\langle\phi\rangle_{\alpha}$ dynamics and of the mean pressure $\left\langle P_{s}\right\rangle_{\alpha}$. In [11 we have identified a nonlinear coupling mechanism of ZF mediated by CTIM. The driving force of ZF was derived, in which the contribution of interchange mode is included. The time evolution of $\left\langle P_{s}\right\rangle_{\alpha}$ obeys the relation:

$$
\frac{\partial\left\langle P_{s}\right\rangle_{\alpha}}{\partial t}=-\left\langle\left[\delta \phi, \delta P_{s}\right]\right\rangle_{\alpha}-\frac{2}{3} \partial_{\psi}\left\langle\left[\bar{\triangle} \delta \phi, \partial_{\alpha} \delta M_{s 2}\right]\right\rangle_{\alpha}
$$

indicating that it is the non-adiabacity between $\delta P_{s}$ and $\delta \phi$ which nonlinearly drives a modification of the mean pressure $\left\langle P_{s}\right\rangle_{\alpha}$. Here $\delta M_{s 2}$ represents the fluctuating moment of second-order. An important question that then arises is how Eq. (7) affects the properties of ZFs.

It is possible to connect the time variation of $\langle\phi\rangle_{\alpha}$ of ZFs to the $\delta P_{s}$ fluctuations. Including the electron contribution, a little algebra (see Ref. [1] for more details) leads to:

$$
\begin{gathered}
\frac{\partial\langle\phi\rangle_{\alpha}}{\partial t}=-\frac{3 T_{i}}{8 T_{e} C_{i}}\left[\left\langle\left\{\delta P_{i}, \delta \phi\right\}\right\rangle_{\alpha}-\beta\left\langle\left\{\delta P_{e}, \delta \phi\right\}\right\rangle_{\alpha}\right] \\
+\left\langle\frac{\partial \delta \phi}{\partial \psi} \frac{\partial \delta \phi}{\partial \alpha}\right\rangle_{\alpha}+\frac{T_{i}}{T_{0} C_{i}}\left[\frac{\partial\left\langle\delta Q_{i}\right\rangle_{\alpha}}{\partial \psi}-\beta \frac{\partial\left\langle\delta Q_{e}\right\rangle_{\alpha}}{\partial \psi}\right]
\end{gathered}
$$

assuming that there is no dissipation (i.e. $D \rightarrow 0$ in Eqs. (1) and (2)) and by defining the anticommutator as $\{f, g\}=\frac{\partial f}{\partial \psi} \frac{\partial g}{\partial \alpha}+\frac{\partial f}{\partial \alpha} \frac{\partial g}{\partial \psi}$. The heat flux, for species $s$, is then defined as:

$$
\left\langle\delta Q_{s}\right\rangle_{\alpha}=\int_{0}^{2 \pi} \frac{d \alpha}{2 \pi} \frac{3}{2} \delta P_{s}\left(-\frac{\partial \delta \phi}{\partial \alpha}\right)
$$

In Eq. (8) the second term in the second member denotes twice of the Reynolds tensor, while the anticommutator terms are a straightforward consequence of the turbulence induced by interchange. The third term at rhs of Eq. (8) is linked to the heat flux $\langle\delta Q\rangle_{\alpha}$ defined by Eq. (9). In Eq. (8) $\beta=\frac{m_{e} T_{e}}{m_{i} T_{i}}$. We then recognise two basic effects in the $\langle\phi\rangle_{\alpha}$ dynamics evolution, the standard turbulence -induced Reynolds stress already noted by Diamond and Kim [13] (the first term in the right hand-side member of
Eq. (8) ) and the effect induced by the coupling between CTIMs and CTEMs. It must be pointed out that it is the polarization drift, entering in the quasi-neutrality equation (4) through the term $\bar{\triangle} \phi$, that nonlinearly drives the $\mathrm{ZF}$. Indeed, in the adiabatic case, (i.e. $C_{e} \delta \phi=\delta n_{i}-\delta n_{e}$ ) we have also $\delta P_{i}-\beta \delta P_{e}=\frac{8}{3} C_{i} \delta \phi$, and therefore the last term in Eq. (8) disappears and $\frac{\partial\langle\phi\rangle_{\alpha}}{\partial t} \rightarrow 0$.

The different actors implicated in turbulence. Within the framework of a simplified picture of drift-wave turbulence involving ITG instabilities, many actors (defined as mesoscopic structures) are involved, such as as Kelvin-Helmholtz (KH) driven shear flows, CTIMs and CTEMs (since their major contribution lead to growth of nonlinear streamers) and ZFs. ZFs of different nature exist. CTIMs have an ion diamagnetic branch, their coupling mechanism can take the form of either non resonant (fluid-aspect interchange) or resonant interaction, as noted in ref. [12. In order to investigate the role played by the different actors in turbulence, it is interesting to consider a simplified picture provided by the "predator prey" model shown in ref. [14 17. Based on a description of the coupled drift-wave ZF turbulence, the model takes into account a population of drift-wave quanta (acting as prey) growing via the linear ITG instability and feeds ZF (as predator) through the Reynolds tensor. The turbulence (here restricted to drift-wave turbulence) is then regulated through mean shear flow introduced in the form of a velocity term of type $V \sim N^{2}$ where $N$ plays the role of a temperature gradient $\left(N=L_{T} \frac{\nabla T}{T}\right)$. The low-high transition (LH) in ref. [17] is thus based on four quantities: the drift-wave turbulence level $E=\left(\frac{\delta n}{n_{0}}\right)^{2}$ (where $\delta n$ represents density fluctuations), $N$ the $\mathrm{ZF}$ velocity $V_{Z F, R}$ and the mean shear flow on which it intrinsically depends. More recently Zhu et al in [18] have extended the model by introducing a new actor they have called the "streamer" and which can also be interpreted as the streamer-induced zonal flow velocity $V_{Z F, S}$. Thus the model encompasses five terms: $N, E, U_{Z F ; R}=V_{Z F, R}^{2}, U_{Z F, S}=V_{Z F, S}^{2}$ and $V=d N^{2}$. The model introduces also the heating rate $Q$ which is a control parameter of the system. Thus the (Reynolds stress- driven) ZF, $U_{Z F, R}$, and the (streamerdriven) counterpart denoted $U_{Z F, S}$ are indirectly coupled to each other through the evolving temperature gradient and the micro-turbulence level. Following the normalisation introduced in ref. [18] (with constants of the models which can be explicitly found in [19]) the normalised predator- prey system writes

$$
\begin{gathered}
\frac{d E}{d t}=\left(N-N^{4}-E-U_{Z F, R}-U_{Z F, S}\right) E \\
\frac{d U_{Z F, R}}{d t}=\mu_{1}\left(\frac{E}{1+\xi N^{4}}-\eta_{1}\right) U_{Z F, R}
\end{gathered}
$$




$$
\begin{gathered}
\frac{d U_{Z F, S}}{d t}=Q \mu_{2}\left(\frac{E}{1+\xi N^{4}}+a N E-\eta_{2}\right) U_{Z F, S} \\
\frac{d N}{d t}=-(\rho+\sigma E) N+Q
\end{gathered}
$$

Eq. (12) corresponds to the modification of the nature of the ZF induced by the backreaction of the streamers instead of the Reynolds stress. The first and second terms on the right-hand side of Eq. (12) illustrate the generation of $U_{Z F, S}$ by coupling with the Reynolds stress and the resonant character of the instability, while the third term corresponds to a damping term. Here the resonant character is assumed to disappear when $N$ tends to zero and when the heat rate $Q$ tends to zero (leading to an adiabatic state). Thus in the simplified predator- prey picture of the LH transition, only three main actors are usually considered: $E, N$ and the zonal flow (the shear flow effect being taken into account through the $N^{4}$ term), the latter possessing two different natures with its low-frequency and zero- frequency components. We focus here on the impact of the low-frequency oscillating $\mathrm{ZF}$ on the predator- prey model.

Fig. 1 displays the numerical results when solving the predator- prey system of Eqs. (10) to (13). The normalized parameters are $\xi=0.35, \eta_{1}=0.08250, \eta_{2}=0.0830$, $\sigma=0.45, \mu_{1}=35, \mu_{2}=19, \rho=0.45$ and $Q=0.4475$. Here initially $N=1$ and the system starts from small perturbations of $U_{Z F, R}=10^{-3}$, of $U_{Z F, S}=10^{-3}$ and from a turbulence fluctuation level of $E=0.10$. For a three-field system (i.e. with $\mu_{2}=0$ and $a=0$ ), it has been shown in ref. [20] that the fixed point undergoes a Hopf bifurcation into a limit cycle in which the ZF activity comes in bursts. We have recovered here this aspect.. A slight increase of $a$ gives rise to strong nonlinear oscillations, in which the nature of the zonal flow has been strongly modified: $U_{Z F, R} \rightarrow 0$ while, with the increasing of the DW turbulence amplitude, $U_{Z F, S}$ is generated and amplified, which provides an additional suppression of the DW turbulence. It should be emphasised that the transition $\left(U_{Z F, R} \rightarrow U_{Z F, S}\right)$ takes place even for a null value of $a$ (but now at later time for $t \sim 3000$ ). Thus the term $a N E$ in (12) leads just to an acceleration towards the transition, a phenomenon somewhat similar to the "phase locking" met in the resonant mechanism.

Resonance aspect of CTIM-CTEM coupling. -

In Ref. 12 we have shown that the oscillatory (i.e. streamer- induced) nature of ZFs is sensitive to the resonant amplification via parametric scattering. In particular the dispersion relation (by considering the potential perturbation mode in the form $\delta \phi_{n}(\psi) e^{i(n \alpha-\omega t)}$ and by assuming adiabatic electrons) can be obtained in the following form:
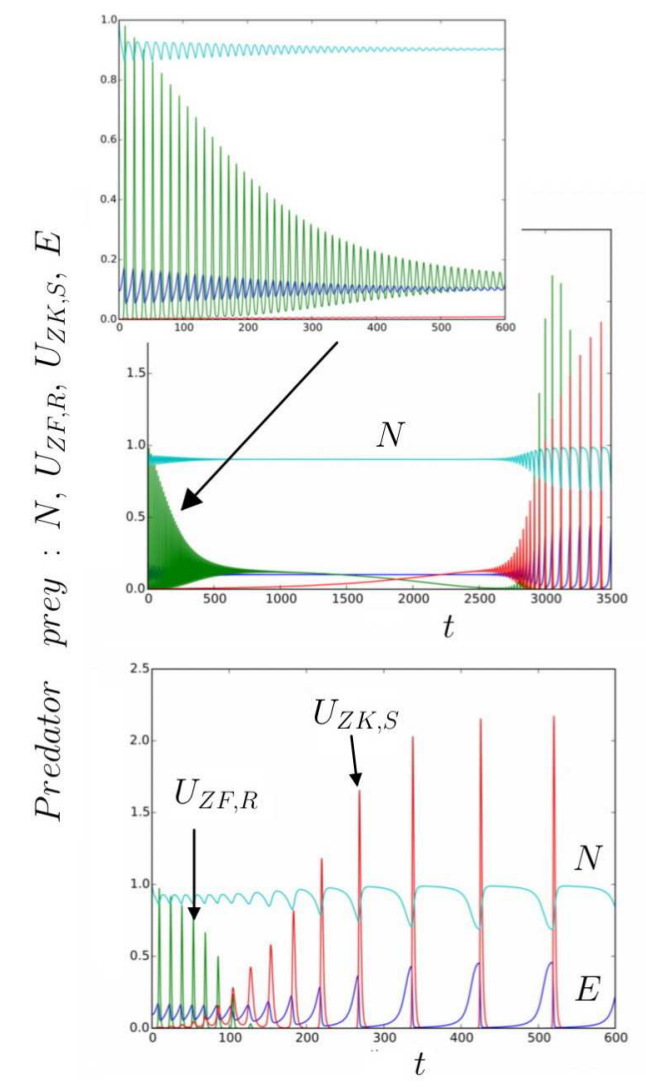

Fig. 1: Time evolution of the different actors implicated in the predator- prey model given in Eqs (16) to (19): the zonal flow components: $U_{Z F, R}$ induced by the Reynolds tensor (in green) and $U_{Z F, S}$ induced by the streamers (in red), the ITG-type temperature gradient $N$ (in blue) and the drift-wave turbulence $E=\left(\delta n / n_{0}\right)^{2}$ (in purple). On top panel, for $a=0$, the fixed point undergoes a Hopf bifurcation into a limit cycle in which $U_{Z F, R}$ activity comes in bursts. On bottom panel, now for $a \neq$ $0, U_{Z F, R}$ tends to zero. Now the component $U_{Z F, S}$ becomes the dominant mode.

$$
C_{e} \delta \phi_{n}=\left\langle\frac{n \triangle \tau e^{-E}\left(E-\frac{3}{2}\right)}{E-\frac{\omega}{n \bar{\omega}_{d}(\kappa)}} J_{0} \delta \phi_{n}\right\rangle_{\kappa, E}
$$

where

$$
\langle.\rangle_{\kappa, E}=\frac{2}{\sqrt{\pi}} \int_{0}^{+\infty} d E \sqrt{E} \int_{0}^{1} d \kappa \kappa K(\kappa) .
$$

and with the usual prescription on the imaginary part of $\omega$. In the linear regime, the linear part of Eq. (14) must cancel exactly for the marginal solution, which implies that the dispersion relation for CTIMs is indeed given by $\omega_{C T I M}=\frac{3}{2} n \omega_{d}(\kappa)$. However as first indicated in ref. 21] a better convergence of the expansion for the pole's calculation can be obtained by introducing a development around $\omega-\frac{5}{2} n \omega_{d}$ leading to an estimation of collisionless CTIM frequency of: 


$$
\omega_{C T I M}=\frac{5}{2} n \omega_{d}(\kappa)+\frac{\sqrt{2 \varepsilon} \omega_{e}^{*}}{2(1+\tau) \rho_{c i}^{2} k_{\psi i}^{2}}
$$

where $\omega_{e}^{*}<0$ is the electron diamagnetic frequency (the last term right-hand side has a value close to $\left.n \omega_{d}(\kappa)\right)$. The resulting linear frequency is $\omega_{C T I M} \simeq \frac{3}{2} n \omega_{d 0}=1.5 \omega_{d 0}$ for a toroidal number of $n=1$ and for a deeply trapped ion population $\kappa \simeq 0$. CTIMs can now resonate with precessing electrons in the opposite direction. The resulting three-wave coupling mechanism involves the decay of a resonant CTIM into CTEM and a zonal flow. The matching conditions are

$$
\omega_{C T I M}=-\omega_{C T E M}+\omega_{Z F, S} \quad \text { and } \quad n=n+0
$$

We have

$$
\omega_{C T E M} \simeq-\frac{5}{2} n \omega_{d}(\kappa) \frac{T_{e}}{T_{0}}+\frac{\sqrt{2 \varepsilon} \omega_{e}^{*}}{2(1+\tau) \rho_{s e}^{2} k_{\psi e}^{2}}
$$

or equivalently $\omega_{C T E M} \simeq-\frac{3}{2} n \omega_{d}(\kappa) \frac{T_{e}}{T_{0}}$, which is close to $\frac{-\omega_{C T I M}}{2}$ for an electron temperature of $T_{e}=0.5 T_{0}$ (and $\left.T_{i}=T_{0}\right)$. Thus we expect the growth of a resonant $\mathrm{ZF}$ at the frequency $\omega_{Z F, S}=\omega_{C T I M}+\omega_{C T E M} \simeq 1.5 \omega_{d 0}-$ $0.75 \omega_{d 0}$, i.e. $\omega_{Z F, S} \simeq 0.75 \omega_{d 0}$.

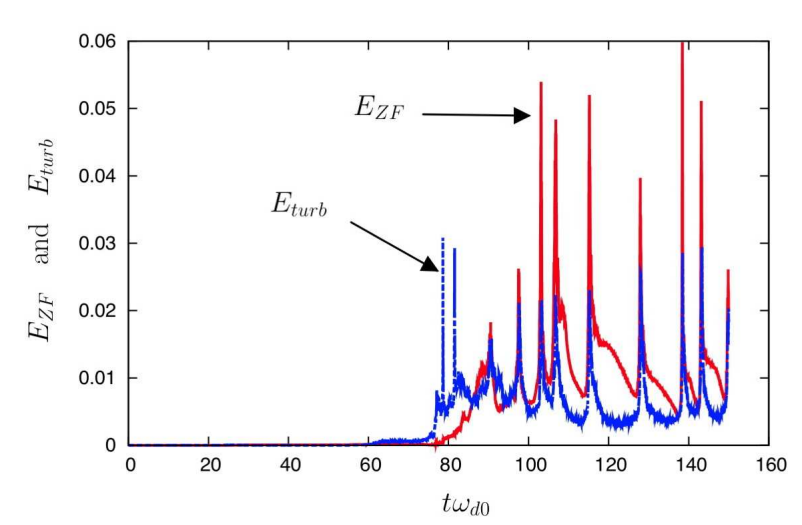

Fig. 2: The corresponding temporal evolution of the turbulent energy $\left(E_{\text {turb }}\right)$ and of the zonal flow energy $\left(E_{Z F}\right)$. In the initial step (for $0 \leq t \omega_{d 0} \leq 90$ ) the micro-turbulence is driven by gradients in pressure. These CTIM and CTEM components constitute the energy source for the growth of a series of poloidal flow in bursts. The following abrupt growth of ZF is considered to be the onset of the parametric coupling between trapped particle modes. The ZF is generated and back reacts upon turbulence which strongly decreases.

Our kinetic trapped particle model is given by a set of $N_{\kappa} N_{E}=16 \times 128$ values in pitch-angle and energy, each value corresponding to a system of Eqs. (1) and (2), coupled together through the quasi-neutrality equation (4). A first simulation was carried out (with both mobile trapped ions and electrons) with an ion temperature gradient of $\triangle \tau=0.90$ chosen above the threshold of the ITG instability given by $\triangle \tau_{s}=\frac{C_{e}}{1-\frac{3}{4} \delta_{b i}^{2}+\frac{15}{64} \delta_{b i}^{4}} \simeq 0.5023$ for $C_{e}=0.50$ and a banana width of $\frac{\delta_{b i}}{\Delta \psi}=\frac{\delta_{b e}}{\Delta \psi} \simeq 0.0936$. We choose a Larmor radius of $\frac{\rho_{s}}{\Delta \psi}=\frac{\rho_{s}}{\Delta \psi}=0.01$ and a magnetic shear of $s=\frac{r_{0}}{q_{0}}\left(\frac{d q}{d r}\right)_{0}=0.8$, well inside the region of the ITG instability. The phase space sampling is $N_{\psi}=128$ by $N_{\alpha}=256$. The time step is $\Delta t \omega_{d 0}=0.0075$ and we have chosen $C_{i} \simeq 0.61$ for the polarization term in order to excite a strong coupling. This simulation has been carried out with 2048 processors (each processor using 8 Open-MP tasks, i.e. a total of 16384 cores on the BlueGene of the IDRIS center) using the code semi-lagrangian TERESA code [25], by referring to one of its numerical parallel implementations.

The total energy can be decomposed into three distinct parts, the kinetic energy $E_{K i n, s}$ for each species denoted $s$, the zonal flow contribution denoted by $E_{Z F}$ and the turbulence contribution $E_{\text {turb }}$ defined respectively by:

$$
E_{K i n, s}=\iint \frac{d \alpha}{2 \pi} d \psi \Theta_{s}
$$

where we have introduced the kinetic energy density in the form:

$$
\begin{gathered}
\Theta_{s}=\psi \omega_{d 0}\left\langle\bar{\omega}_{d}(\kappa ; s) E_{s} f_{\kappa, E ; s}\right\rangle_{\kappa, E_{s}} \\
E_{Z F} \simeq \frac{1}{2} C_{i} \delta_{b i}^{2} \iint \frac{d \alpha}{2 \pi} d \psi\left(\frac{\partial\langle\phi\rangle_{\alpha}}{\partial \psi}\right)^{2} \\
E_{\text {turb }}=\iint \frac{d \alpha}{2 \pi} d \psi\left(\frac{1}{2} C_{e} \delta \phi^{2}+\frac{1}{2} C_{i}|\bar{\nabla} \delta \phi|^{2}\right)
\end{gathered}
$$

The resonant CTIM- CTEM coupling is illustrated in Fig. 2 in which we have plotted the time evolution of the (total) ZF energy $E_{Z F}$ given by Eq. (21) superimposed on the turbulence energy $E_{\text {turb }}$ defined in (22).

We get the entire physical picture. It is the initial modification of the equilibrium distribution function that induces a sheared electric field and leads to the weak decrease of kinetic energies for both species, followed by the occurrence of the two first peaks observed in the turbulent energy at time $t \omega_{d 0} \simeq 80$. Then trapped particle modes (both CTIM and CTEM) participate to the turbulent transport. They couple together nonlinearly and in a resonant parametric-type process to screen the induced sheared electric field (triggered by the Reynolds tensor). The latter is this way suppressed and streamers are excited which modify the nature of ZFs. It must be pointed out that the oscillating $\mathrm{ZF}$ component can be excited and its amplitude strongly enhanced by streamers (the resonant component of CTEM) at low frequency close to the drift frequency $\omega_{d 0}$. As the resonant CTEM is excited, corresponding to the mode $n=1$, with a frequency close 
to $\omega_{C T E M} \simeq-\frac{3}{2} n \omega_{d}(\kappa) \frac{T_{e}}{T_{0}} \simeq-0.75 \omega_{d 0}$, the parametric coupling takes place in a nonlinear way (through its second harmonics $-2 \omega_{C T E M}=\omega_{C T I M}=1.50 \omega_{d 0}$, leading to the growth of the ZF component triggered by the polarization effects, as expected by Eq. (21). It must be pointed out that both the turbulent energy and ZF energy exhibit resonant peaks at the same instants. The nonlinear driver of ZF is here governed by the nonlinear streamer described by the difference of the anticommutators $\left\langle\left\{\delta P_{i}, \delta \phi\right\}\right\rangle_{\alpha}-\beta\left\langle\left\{\delta P_{e}, \delta \phi\right\}\right\rangle_{\alpha}$ in (8). Note that the time evolution of ZF exhibits also the beating of frequencies $\omega_{Z F, S}=0.75 \omega_{d 0}$ and $\frac{\sqrt{2 \varepsilon}\left|\omega_{e}^{*}\right|}{2(1+\tau) \rho_{s i}^{2} k_{\psi i}^{2}} \sim \omega_{d 0}$.

Ion transport barrier triggered by the polarization drift. - It was shown experimentally that fusion plasmas exhibits transitions that involve the reduction in turbulent transport through the creation of transport barriers (TBs). Such TBs are radially localised at the edge (H-mode), or in the bulk part of the plasma, where they appear in the core. Both TBs are reported to correlate well with the existence of sheared radial electric fields and are identified by very steep radial profiles of ion or electron temperature (see ref. 24]). More recently, within the context of gyrokinetic simulations [22,23, it was shown that the TB can be triggered by a vorticity source that polarises the plasma. It appears that the dynamics of these TBs exhibits quasi-periodic crashes, which are found to result from the complex interplay between the injection of a polarization source and its backreaction with turbulence. The authors have demonstrated the possibility to design a vorticity (or polarization) source in full gyrokinetic simulations that can modify the distribution in phase space and successfully induces a localised sheared electric field.

In the same spirit, we choose to increase strongly the (normalized) perturbation (Heat) amplitude to 0.15 (which indeed allow to inject initially a heat flux of type (9) (without pertubating the initial ITG instability, the polarization injecting corresponds indeed to a gradient of temperature well below the threshold of the ITG instability and leads thus to a slight modification of the initial equilibrium). As the resonant CTEMs and CTIMs are excited, we observe a fast growth of the ZF linked by the polarization $\left(C_{i} \neq 0\right)$ effects. Here the choice of a ratio of temperature of $\tau=1$ (i.e. $T_{i}=T_{e}=T_{0}$ ) is chosen to reduce the amplitude or resonance and the amplitude of the second resonant peak in order to illustrate the importance of the initial shear flow. This allows a resonant process of the kind decay of the fluid interchange mode into CTIM plus ZF)

$$
\frac{5}{2} n \omega_{d}(\kappa) \frac{T_{i}}{T_{0}}=\frac{3}{2} n \omega_{d}(\kappa) \frac{T_{i}}{T_{0}}+\frac{\sqrt{2 \varepsilon} \omega_{e}^{*}}{2(1+\tau) \rho_{c i}^{2} k_{\psi i}^{2}}
$$

and the resulting coupling $-2 \omega_{C T I M}+\omega_{C T I M} \simeq \omega_{C T E M}$ since $\omega_{C T E M}=-\frac{3}{2} n \omega_{d}(\kappa) \frac{T_{e}}{T_{0}}$ which is also $-\omega_{C T I M}$.

Fig. 3 shows the time evolution of the ion kinetic energy and the corresponding turbulent energy $E_{\text {turb }}$. It is worth
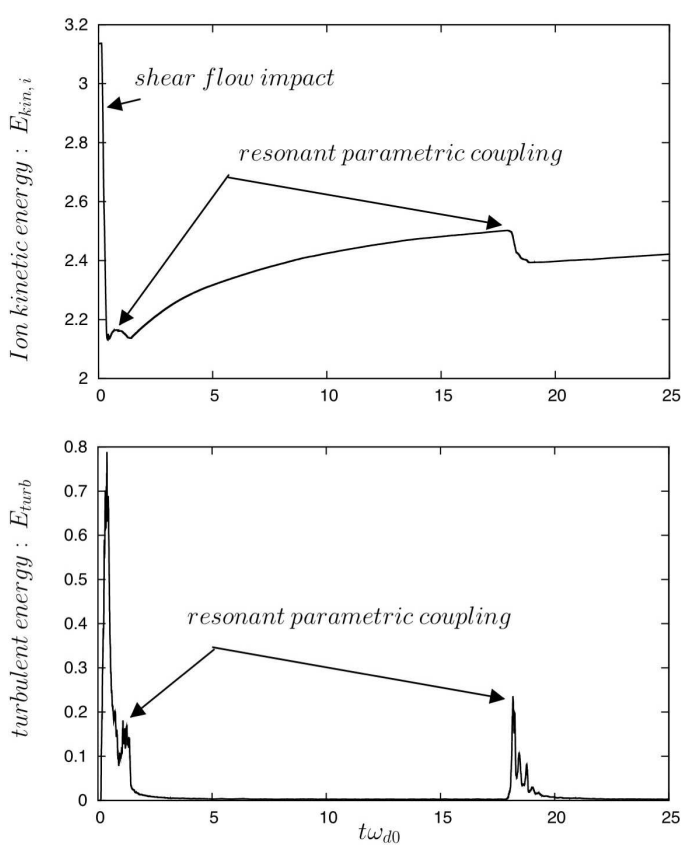

Fig. 3: Temporal evolution of the ion kinetic energy (on top panel) and of the turbulent energy (shown on bottom panel) for the transport barrier formation. Once the sheared flow is formed (first dominant peak on bottom panel), the parametric coupling between trapped particle modes (CTIM plus CTEM) takes places leading to the formation of TB. When shear flow and ZF become negligible, TB collapses and a turbulent burst is occurring.

mentioning that we could also test the resilience of the induced TB, which is built in a self- consistent way. In particular we observe that the barrier is marginally stable and a second weaker burst of turbulence takes place at time $t \omega_{d 0} \simeq 18$. We display in Fig. 4 the system's response (in term of density) to the introduction of a polarization source. CTIM and CTEM participate to the turbulent transport, they couple together nonlinearly in a resonant parametric beating to enhance the $\mathrm{ZF}$ contribution which results in the suppression of turbulence. On top panel in Fig. 4, the level of turbulence is high (just before the formation of $\mathrm{TB}$ ), but the plasma systematically relaxes slowly towards an "equilibrium state" (shown at a given time $t \omega_{d 0} \simeq 16$ ). Once the sheared flow and ZFs become negligible, nothing remains to maintain the stability of TB, which collapses. Then the barrier experiences a first turbulent burst at time $t \omega_{d 0} \simeq 18.25$.

Conclusion . - In this Letter, we have found a new mechanism that can generate a transport barrier in tokamak plasma. We have identified a nonlinear and resonant coupling mechanism of zonal flow mediated by trapped ion and trapped electron modes in the collisionless regime. The role of the shear flow induced by the initial polarization injection in the developping theory of self- sustained 

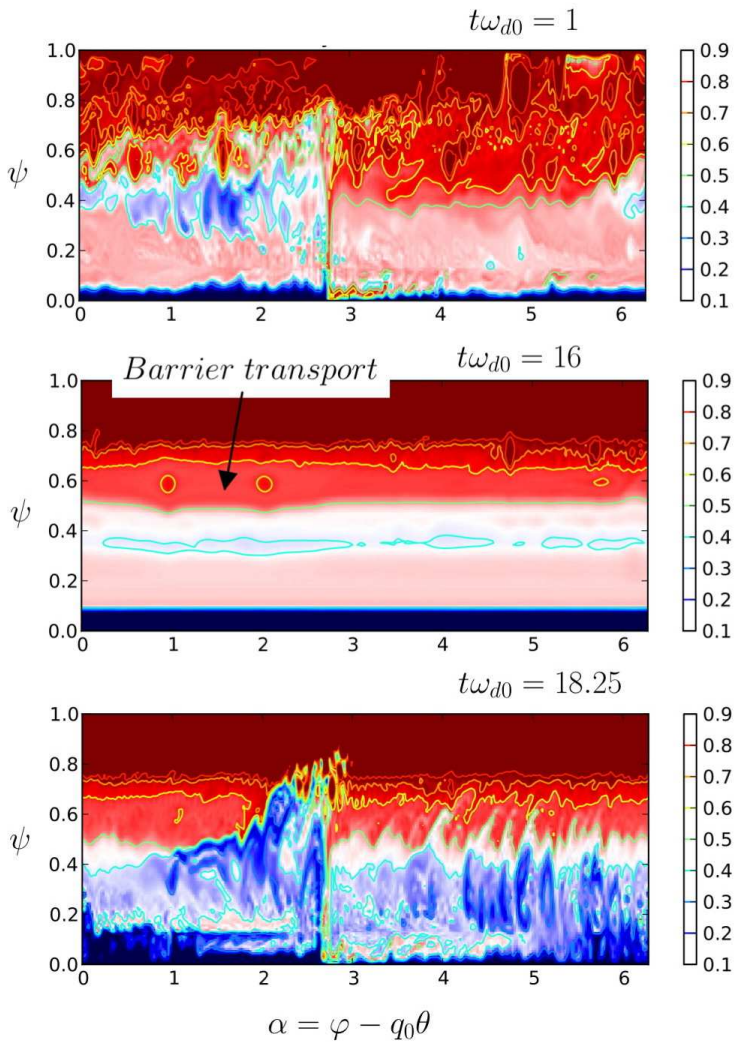

Fig. 4: Phase space illustration in $(\alpha, \psi)$ at three different times showing the formation (on top panel) towards relaxation step (middle panel). On bottom panel, the first crash happens with a certain time delay compared to quenching of the shear flow.

and marginally stable transport barriers will be discussed in a future publication.

Acknowledgment

The authors are indebted to the IDRIS computational center, Orsay, France, for computer time allocation on their computers. This work was granted access to the HPC resources (Grant 2014-2015- 054028) made by GENCI (Grand Equipement National de Calcul Intensif) and project CCUFB/BFHZ FK30-15 funded by the frenchbavarien cooperation centre.

\section{REFERENCES}

[1] BURREL K.H., CARLSTROM T.N., DOYLE E.J., FINKENTHAL D., GOHIL R.J., GROEBNER R.J., HILLIS D.L.,KIM J., MATSUMOTO H., MOYER R.A., OSBORNE T.H., RETTIG C.L., PEEBLES W.A., RHODES T.L., ST JOHN H., STAMBAUGH R.D., WADE M.R., WATKINS J.G. and DIII Team, Plasma Phys. Controlled Fusion 34 (1992) 1859.

[2] WAGNER F. et al, Phys. Rev. Letters 49 (1982) 1408.

[3] WAGNER F. et al, Phys. Rev. Letters 53 (1984) 1453.

[4] DOYLE E.J. ET AL, Nuclear Fusion 47 (2007) S18.
[5] XU G.S., WAN B.N., WANG H.Q., GUO H.Y., ZHAO H.L., LIU A.D., NAULIN V., DIAMOND PH, TYNAN G.R., XU M., CHEN R., JIANG M., LIU P., YAN N., ZHANG W., WANG L., LIU SC, DING S.Y. Phys. Rev. Lett. 107 (2011) 125001.

[6] GUPTA D.K., FONCK R.J., McKEE G.R., SCHLOSSBERG D.J., SHAFER M.W., Phys. Rev. Lett. 97 (2006) 125002.

[7] GHIZZO A., DEL SARTO D., GARBET X., SARAZIN Y., Physics of Plasmas 17 (2010) 092501.

[8] KADOMTSEV B.B., POGUTSE O.P. Nuclear Fusion 11(1971) 67.

[9] KIM K., PARK J.K., BOOZER A.H. Phys. Rev. Lett. 110 (2013) 185004.

[10] KIKUCHI M., AZUMI M., Reviews of Modern Phys. 84 (2012) 1807.

[11] GHIZZO A., PALERMO F. Physics of Plasmas 22 (2015) 082303.

[12] GHIZZO A., PALERMO F. Physics of Plasmas 22 (2015) 082304.

[13] DIAMOND P.H., KIM Y.B. Phys. Fluids B3 (1991) 1626.

[14] MALKOV M.A., DIAMOND P.H., ROSENBLUTH M.N. Physics of Plasmas 8 (2001) 5073.

[15] KIM E.J., DIAMOND P.H., Phys. Rev. Letters 90 (2003) 185006.

[16] KIM E.J., DIAMOND P.H. Phys. Plasmas 10 (2003) 1698.

[17] MALKOV M.A., DIAMOND P.H. Physics of Plasmas 15 (2008) 122301.

[18] ZHU H., CHAPMAN S.C., DENDY R.D. Physics of Plasmas 20 (2013) 042302.

[19] DIAMOND P.H., LIANG Y.M., CARRERAS B.A., TERRY P.W. Phys. Rev. Letters 72 (1999) 2565.

[20] MALKOV M.A., DIAMOND P.H. Physics of Plasmas 15 (2009) 012504.

[21] TANG W.M., ADAM J.C. ROSS D.W. Phys. Fluids 20 (1977) 430.

[22] STRUGAREK A., SARAZIN Y., ZARZOSO D., ABITEBOUL J., BRUN A.S., CARTIER MICHAUD T., DIF PRADALIER, GARBET X., GHENDRIH P.H., GRANGIRARD V. LATU G., PASSERON C., THOMINE O. Plasma Phys. Control. Fusion 55 (2013) 074013.

[23] STRUGAREK A., SARAZIN Y., ZARZOSO D., ABITEBOUL J., BRUN A.S., CARTIER MICHAUD T., DIF PRADALIER, GARBET X., GHENDRIH P.H., GRANGIRARD V. LATU G., PASSERON C., THOMINE O. , Phys. Rev. Letters 111 (2013) 145001.

[24] WOLF R.C. Plasma Phys. Control. Fus. 45 (2003) 1.

[25] CARTIER- MICHAUD T., GHENDRIH P., GRANDGIRARD V., LATU. G. ESAIM Proceedings, 93 (2013) EDP Sciences. 